\title{
Fístula colecistocolónica: abordaje dual endoscópico. Reporte de un caso
}

\author{
Dante Manazzoni, Ignacio Málaga, Cecilia Curvale, Martín Guidi, Julio de María, Raúl Matano \\ Hospital de alta complejidad El Cruce. Florencio Varela, Provincia de Buenos Aires, Argentina.
}

Acta Gastroenterol Latinoam 2020;50(3):360-364

Recibido: 07/05/2018 / Aceptado: 19/02/2020 / Publicado online: 28/09/2020

\section{Resumen}

La fistula colecistocolónica es una complicación poco habitual y tardía de la litiasis vesicular. Los sintomas suelen ser inespecificos y la mayoría se diagnóstica de forma intraoperatoria. Una mujer de 75 años, tabaquista, con un antecedente de estenosis aórtica severa, consultó por una diarrea de 6 meses de evolución asociada a la pérdida de peso y a la alteración del coagulograma y aerobilia en una ecografía de control. Ante la sospecha clínica de una fistula colecistocolónica se le solicitó una tomografía de abdomen y una colangioresonancia en las que se documentó su presencia. Dadas las comorbilidades de la paciente, se optó por un tratamiento no quirúrgico. Este consistió en una colangiografía retrógrada endoscópica con la extracción de la litiasis coledociana y la colocación de un stent metálico autoexpansible totalmente cubierto, asociado a una videocolonoscopía con cierre del orificio colónico mediante un clip. La paciente evolucionó de forma favorable desde el segundo día luego del procedimiento. En los tres meses posteriores de seguimiento no presentó diarreas y ha recuperado la totalidad del peso perdido. Dado lo inusual del caso y la trascendencia del diagnóstico

Correspondencia: Cecilia Curvale

Avenida Calchaqui 5401 (C.P.: 1888). Florencio Varela, Provincia de Buenos Aires, Argentina

Tel.: (+54)01142109000

Correo electrónico: cecicurvale@hotmail.com preoperatorio, el cual nos permitió realizar un tratamiento minimamente invasivo, es que creemos útil su reporte. Hacemos especial énfasis en la tríada de la aerobilia, la alteración de la concentración de protrombina y la diarrea crónica que nos permitió sospechar el diagnóstico.

Palabras claves. Fistula colecistocolónica, neumobilia, diarrea crónica, déficit de vitamina $K$, tratamiento endoscópico.

\section{Cholecistocolonic fistula: dual endoscopic approach. Case report}

\section{Summary}

Cholecystocolonic fistula is an unusual and late complication of cholelithiasis. The symptoms usually are nonspecific and most cases are diagnosed intraoperatively. Our patient was a female, 75 years old, smoker, with a severe aortic stenosis history. She had a 6-month evolution diarrhea associated with weight loss, coagulopathy and aerobilia in abdominal ultrasound. On behalf of the clinic manifestations, cholecystocolonic fistula was suspected so an abdominal computer tomography scan and a cholangioresonance confirmed this suspicion. Given the patient comorbidities, we decided not to perform a surgical approach. An endoscopic retrograde cholangiography with extraction of choledocolithiasis and placement of a fully covered self-expanding metallic stent was associated with a video colonoscopy and closure of the colonic fistula with a clip device. The patient had a favourable evolution and was discharged on the second day post-procedure. After 3 months of follow-up, she did not present diarrhea and has recovered her weight loss and nutritional status. Given the unusual nature of the case and the significance of the preoperative diagnosis, which allowed us to carry out 
a minimally invasive treatment, we believe that its report is useful. We place special emphasis on the aerobic triad, alteration of the prothrombin concentration and chronic diarrhea that allowed us to suspect the diagnosis.

Key words. Cholecistocolonic fistula, pneumobilia, chronic diarrhea, vitamin $K$ deficiency, endoscopic treatment.

\section{Abreviaturas}

FCC: Fistula colecistocolónica.

FEB: Fistula entero biliar.

CPRE: Colangiografía retrograda endoscópica.

VEDA: Videoendoscopía digestiva alta.

VCC: Videocolonoscopía.

CP: Concentración de protrombina.

TC: Tomografía computada.

CRMN: Colangioresonancia magnética nuclear.

EPT: Esfinteropapilotomía.

SEMS: Stent metálico autoexpansible.

\section{Introducción}

La fístula colecistocolónica (FCC) es una complicación poco habitual y tardía de la litiasis vesicular. Es la segunda causa más frecuente dentro de las fístulas enterobiliares (FEB) luego de las colecistoduodenales. Los síntomas suelen ser mínimos o inespecíficos. Los métodos por imágenes carecen de sensibilidad para evidenciar la fístula por lo que el diagnóstico suele ser intraoperatorio, encontrándose en 1 de cada 1000 colecistectomías. ${ }^{1}$ Si bien el tratamiento de elección no está claramente definido, su resolución suele ser quirúrgica. La cirugía genera un escenario desafiante, debido a su complejidad y la de los pacientes, que suelen ser añosos y con múltiples comorbilidades. ${ }^{1}$

Figura 1. Tomografía computada. Corte axial y coronal
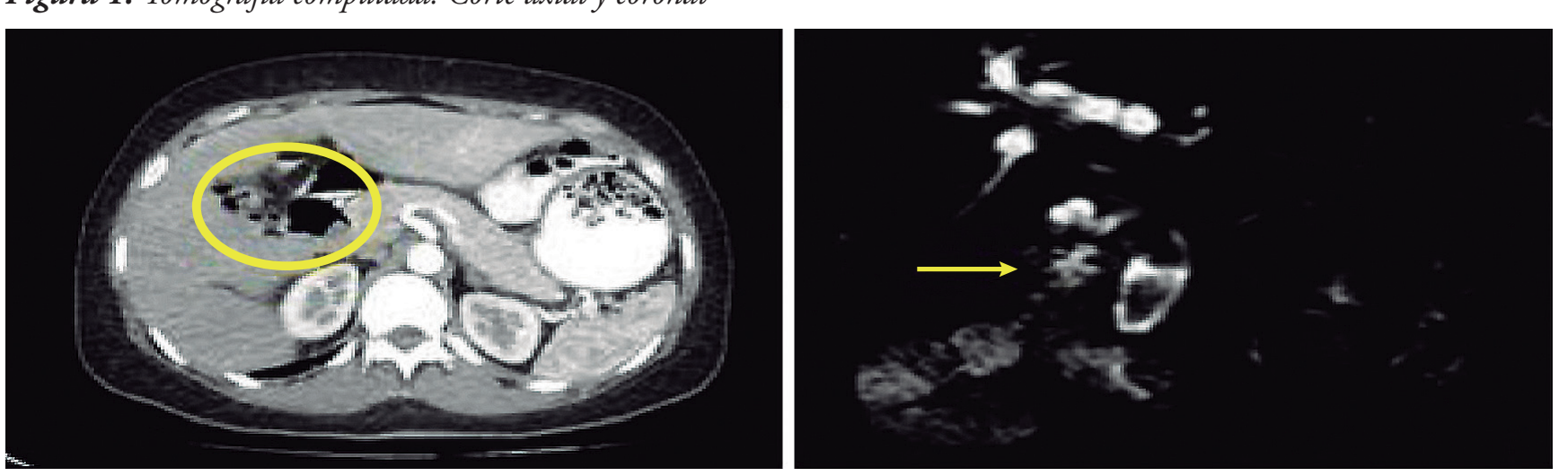

TC: Se observa el colédoco dilatado con un lito en su interior con la vesícula contraída en cercanías a un asa colónica (círculo amarillo). Puede verse también

probable trayecto fistuloso entre el lecho vesicular y el colon (flecha amarilla).
Por lo antes mencionado, decidimos presentar este caso de una mujer de 75 años en el que la combinación de los síntomas, las imágenes y el laboratorio permitieron hacer el diagnóstico preoperatorio de una FCC, lo cual posibilitó la aplicación de un tratamiento endoscópico.

\section{Caso clínico}

Una mujer de 75 años consultó por una diarrea de 6 meses de evolución y la pérdida de 5 kilos. El cuadro había iniciado con 4 a 6 deposiciones por día, líquidas, "verdosas", no asociadas a la ingesta y de predominio diurno. Nunca presentó dolor abdominal, hematoquecia, melena ni otros síntomas. El examen físico fue normal. Como antecedentes de relevancia presentaba estenosis aórtica severa, hipertensión arterial y tabaquismo (40 paq./año).

En el laboratorio el único hallazgo de relevancia fue una concentración de protrombina (CP) de 57\% (VN superior a 70). El resto de la rutina, incluyendo los anticuerpos para la enfermedad celíaca, el perfil tiroideo y los estudios de la materia fecal, fue normal. También se había realizado una videoendoscopía digestiva alta (VEDA) y una videocolonoscopía (VCC) con biopsias

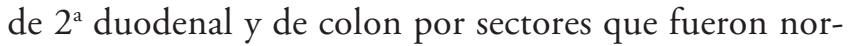
males (descartando malabsorción y colitis microscópica). En la ecografía abdominal se destacó la presencia de aerobilia.

Ante la tríada de la diarrea crónica, la alteración de la CP y la aerobilia, se sospechó de una FCC, motivo por el cual se solicitó una tomografía (TC) de abdomen con contraste oral y endovenoso que luego se complementó con una colangioresonancia (CRMN). En la misma se observó una litiasis coledociana gigante, la vesícula contraída y un trayecto fistuloso entre el lecho vesicular y el ángulo hepático del colon (Figura 1). 
Se evaluó la paciente en conjunto con el equipo de cirugía y se decidió, dadas las comorbilidades, realizar un tratamiento endoscópico. Se planteó un abordaje combinado, a través de la realización de una colangiografía retrograda endoscópica (CPRE) para resolver la coledocolitiasis, identificar la fístula y colocar un stent metálico autoexpansible totalmente cubierto (SEMS) y posteriormente una VCC para intentar identificar el orificio colónico de la fístula con su cierre (Figura 2).
En la imagen se observa la vía biliar principal dilatada con un lito de gran tamaño en el tercio medio del colédoco. Se logra apreciar el pasaje de contraste a través del trayecto fistuloso hacia el colon ascendente (Figura 3).

Se le realiza la esfinteropapilotomía (EPT) y se la dilata con un balón de $15 \mathrm{~mm}$.

Se le realiza la litotripsia mecánica y se extraen múltiples fragmentos con el balón extractor y la canastilla.

Figura 2. Colangioresonancia magnética nuclear y esquema

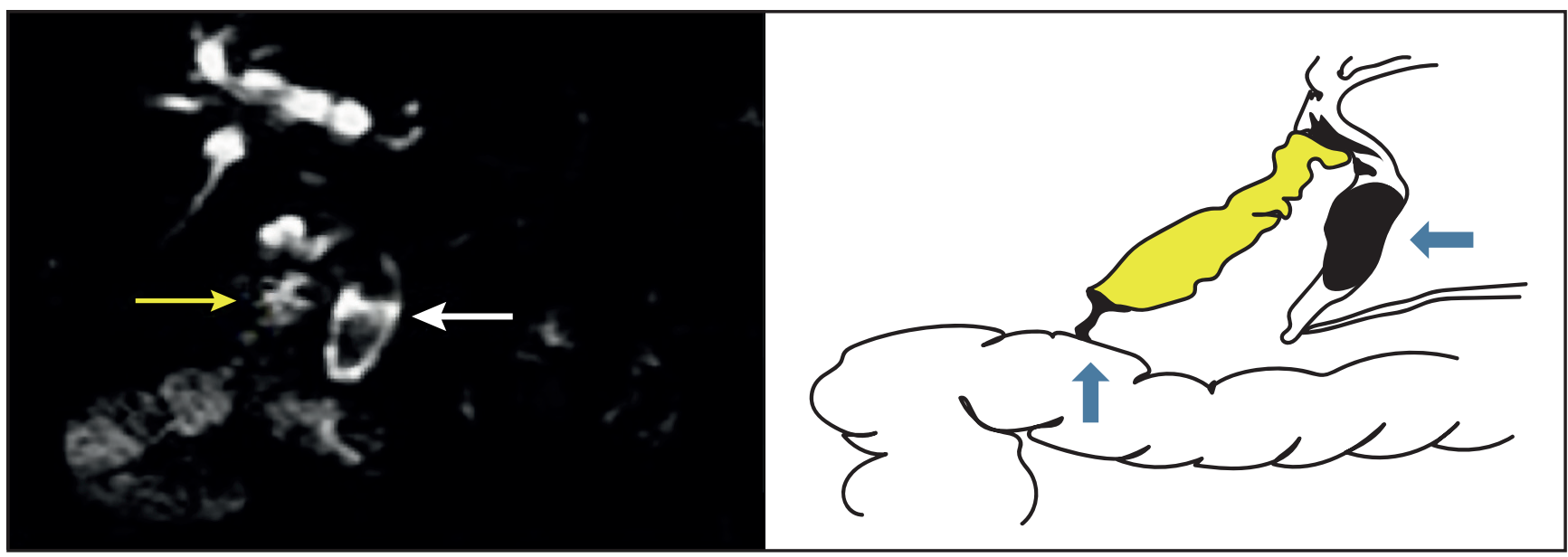

Se observa a la izquierda en la CRMN el trayecto fistuloso (flecha amarilla) y el colédoco dilatado con litiasis coledociana (flecha blanca). A la derecha está presente una representación del trayecto fistuloso (verde) y el doble abordaje, resolviendo la litiasis coledociana y el orificio colónico de la fistula (flechas azules).

\section{Figura 3. Colangiografía endoscópica retrógrada}

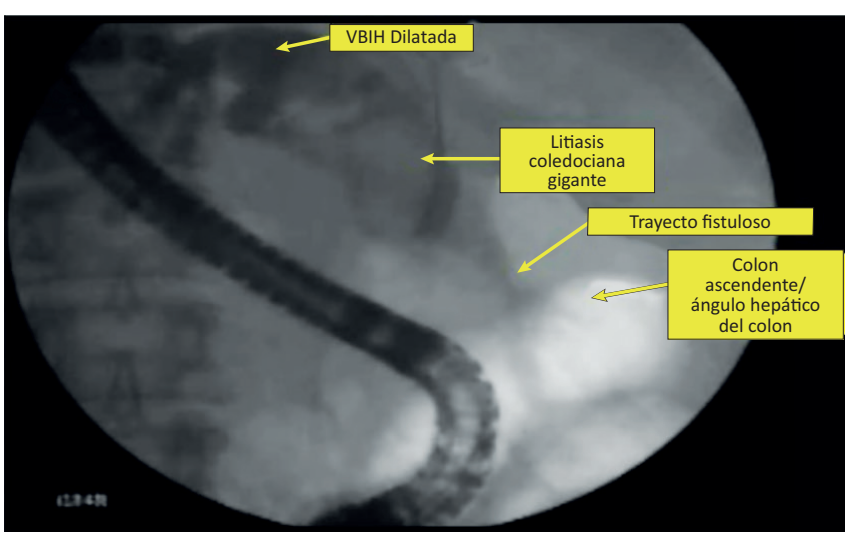

Se coloca un SEMS de $10 \mathrm{~mm}$ por $60 \mathrm{~mm}$. Previamente, y a través de un catéter, se instila azul de metileno en la vía biliar para ayudar a identificar el orificio colónico de la fístula. En la VCC se observan restos de azul de metileno en colon ascendente y se logra identificar el orificio fistuloso donde se colocan dos clips hemostáticos.
La paciente evolucionó favorablemente. 48 horas después del procedimiento cedieron las diarreas y en los tres meses posteriores de seguimiento ha recuperado su peso y persiste asintomática.

\section{Discusión}

Las FCC se encuentran en el 0,06-0,14\% de las colecistectomías. ${ }^{1} \mathrm{Su}$ etiología puede estar relacionada a un proceso inflamatorio de la vesícula biliar, de larga duración, causado por cálculos. Durante este proceso la inflamación crónica, asociada a un aumento de presión dentro de la vesícula, generaría un área de isquemia en la pared vesicular y colónica constituyéndose la FCC. ${ }^{2}$

Los síntomas de la FCC suelen ser mínimos e inespecíficos, siendo la diarrea crónica el más frecuente. En la serie reportada por Hession et al., ${ }^{3}$ alcanza el $71 \%$ de los casos. Esto se debe a una alteración de la circulación entero biliar de las sales biliares que al no absorberse en el íleon distal llegan al colon y estimulan la secreción de agua y electrolitos por la mucosa colónica. Las sales bilia- 
res son fundamentales para la formación de micelas, las cuales permiten la absorción de las grasas y las vitaminas liposolubles. ${ }^{5,6}$

Esto explica por qué se produce una mala absorción de las vitaminas A, D, E y K. El déficit de esta última se objetiva mediante un análisis alterado del $\mathrm{CP} .{ }^{4}$

De forma llamativa, el dolor en el hipocondrio derecho rara vez se encuentra presente en los pacientes con FCC. Aunque se podría suponer que una FCC tiene un alto potencial de infección de la vesícula y el sistema biliar, la ictericia y la fiebre, marcas típicas de colangitis, rara vez se informan como síntomas del inicio en una FCC. ${ }^{2,4}$

Según la revisión realizada por Chowbey et al., solo el 7,9\% de las FCC se diagnostica en instancia preoperatoria. ${ }^{1}$ Esta baja tasa de detección se debe a la poca especificidad de los síntomas y a la baja sensibilidad de los métodos diagnósticos. El colon por enema parece ser el mejor método para arribar al diagnóstico, pero carece de sensibilidad. Hay reportes aislados de los diagnósticos realizados mediante TC de abdomen, ${ }^{7}$ pero esta no se utiliza de forma sistemática.

En el caso de nuestra paciente, la aerobilia observada en la ecografía abdominal fue determinante ya que, junto con los datos de laboratorio y la clínica, nos hizo sospechar la presencia de una FCC que pudo ser confirmada mediante la combinación de la TC de abdomen y la CRMN. La tríada de la aerobilia, la alteración del coagulograma y la diarrea crónica fue propuesta como patognomónica por Savvidou et al..$^{5}$ en la presentación de un caso.

Con respecto al tratamiento, existen diversos enfoques, desde grandes resecciones colónicas y del lecho vesicular hasta un manejo conservador mediante CPRE. Durante los últimos años y en pacientes sin complicaciones por la FCC, la tendencia gira en torno a tratamientos menos invasivos. ${ }^{1,2,8}$

Algunos autores proponen la CPRE como un manejo inicial para los pacientes en mal estado general o con comorbilidades ya que la EPT con la extracción de la litiasis coledociana reduciría la presión en la vesícula y cerraría la fístula con mínimos riesgos para el paciente. ${ }^{1}$ Según nuestro conocimiento, solo se han reportado cuatro casos tratados de esta manera. El primero de ellos es en un paciente de 89 años que presentaba una alteración de la CP y litiasis coledociana. El diagnóstico se le realizó durante la CPRE y la EPT, con la extracción de los litos resolvió la FCC. ${ }^{8}$ El segundo caso, publicado por Marshall et al., fue en un paciente de 86 años con un diagnóstico preoperatorio de una FCC mediante un colon por enema. El paciente tenía una diarrea crónica y pérdida de peso. Se le realizó una EPT y se le colocaron dos stents plásticos de 7 Fr debido a la imposibilidad de retirar los litos de la vía biliar. El paciente mejoró los síntomas al segundo día después del procedimiento. ${ }^{9}$ El tercer caso fue sobre una mujer de 72 años con una historia de dolor abdominal en el hipocondrio derecho, una diarrea crónica y pérdida de peso. Este caso se resolvió con una EPT y con la extracción de la litiasis coledociana. ${ }^{10}$ En el cuarto caso, del cual se cuenta solo con el abstract, se relata el caso de un paciente masculino de 87 años que se le resolvió la fístula solo con la realización de la EPT. ${ }^{11}$ Nuestro caso sería el quinto que se resuelve de manera endoscópica y el primero en el que se coloca un SEMS para cerrar el orificio biliar de la fístula.

Debemos destacar que los pacientes con la vesícula no resultarían de riesgo para esta técnica ya que no se ha evidenciado un incremento en las tasas de colecistitis asociada a la utilización de stents metálicos cubiertos en esta población. ${ }^{12,13}$ Por el contrario, si el tratamiento elegido es quirúrgico se presentan cuestiones netamente relacionadas con la técnica como la necesidad o no de una colostomía o la factibilidad del cierre de la FCC mediante una laparoscopia. Hasta principios de los años noventa la laparoscopia estaba contraindicada en las FCC, sin embargo, en la actualidad el enfoque inicial es laparoscópico. ${ }^{1,14}$ Aunque se postula la viabilidad del procedimiento, también se reportan largos tiempos operatorios y un número considerable de conversiones. Un estudio multicéntrico informa la conversión temprana en el 55\% de los pacientes. ${ }^{14}$ Los autores concluyen que debería evaluarse cada caso en particular para buscar el tratamiento menos invasivo posible.

En conclusión, reportamos un caso infrecuente de la FCC, resuelto mediante CPRE gracias al diagnóstico preoperatorio. Consideramos de suma importancia tener presente la tríada de la diarrea crónica, la alteración del CP, la neumobilia y su asociación con la FCC. El diagnóstico preoperatorio permite, en este grupo de pacientes que suelen ser añosos y con múltiples comorbilidades, optar por un tratamiento endoscópico con mínimos riesgos.

Conflicto de intereses. Ninguno de los autores tiene conflictos de intereses.

Sostén financiero. Este manuscrito no contó con financiación.

\section{Referencias}

1. Chowbey PK, Bandyopadhyay SK, Sharma A, Khullar R, Soni V, Baijal M. Laparoscopic management of cholecystoenteric fistulas. J Laparoendosc Adv Surg Tech A 2006; 16 (5): 467-472. Disponible en doi: 10.1089/lap.2006.16.467. 
2. Costi R, Randone B, Violi V, Scatton O, Sarli L, Soubrane O, Dousset B, Montariol T. Cholecystocolonic fistula: facts and myths. A review of the 231 published cases. Journal of Hepatobiliary Pancreat Surg 2009; 16 (1): 8-18. Disponible en doi: 10.1007/s00534-008-0014-1.

3. Gora N, Singh A, Jain S, Parihar US, Bhutra S. Spontaneous Cholecystocolic Fistula: Case Report. Journal of Clinical and Diagnostic Research 2014; 8 (3): 164-165. Disponible en doi: 10.7860/ JCDR/2014/7298.4149.

4. Hession PR, Rawlinson J, Hall JR, Keating JP, Guyer PB. The clinical and radiological features of cholecystocolic fistulae. Br J Radiol 1996; 69 (825): 804-809. Disponible en doi: 10.1259/00071285-69-825-804.

5. Savvidou S, Goulis J, Gantzarou A, Ilonidis G. Pneumobilia, chronic diarrhea, vitamin $\mathrm{K}$ malabsorption: A pathognomonic triad for cholecystocolonic fistulas. World J Gastroenterol 2009; 15 (32): 4077-4082. Disponible en doi: 10.3748/wjg.15.4077.

6. Munene G, Graham, JA, Holt RW, Johnson LB, Marshall Jr HP. Biliary-colonic fistula: a case report and literature review. Am Surg 2006; 72 (4): 347-350.

7. Singh AK, Gervais D, Mueller P. Cholecystocolonic fistula: serial CT imaging features. Emerg Radiol 2004; 10 (6): 301-302. Disponible en doi: 10.1007/s10140-004-0353-4.

8. Marshall T, Kamalvand K, Cairns SR. Endoscopic treatment of biliary enteric fistula. Br Med J 1990; 300 (6733): 1176. Disponible en https:/www.ncbi.nlm.nih.gov/pmc/articles/PMC1662922/ pdf/bmj00177-0032.pdf. Fecha de consulta: 24 de abril de 2018.
9. Goldberg RI, Phillips RS, Barkin JS. Spontaneous cholecystocolonic fistula treated by endoscopic sphincterotomy. Gastrointest Endosc 1988; 34 (1): 55-56. Disponible en doi: 10.1016/S00165107(88)71232-8.

10. Ahmed Ali A, Gompertz H. Cholecysto-colonic fistula; a rare case. Libyan J Med 2007; 2 (3): 142-143. Disponible en doi: 10.4176/070324.

11. Bannister P, Bennett C, Denyer ME. Spontaneous choledochalcolonic fistula treated by duodenoscopic sphincterotomy. Br Med J (Clin Res Ed) 1984; 289 (6452): 1114. Disponible en doi: 10.1136/bmj.289.6452.1114.

12. Saleem A, Leggett CL, Murad MH, Baron TH. Meta-analysis of randomized trials comparing the patency of covered and uncovered self-expandable metal stents for palliation of distal malignant bile duct obstruction. Gastrointest Endosc 2011; 74 (2): 321-327.

13. Curvale C, Guidi M, Hwang H, De María J, Matanó R. Resolution of complex choledocholithiasis with removable metal stent. A case report. Rev Gastroenterol Mex 2016; 81 (1): 56-58. Disponible en doi: 10.1016/j.rgmx.2015.05.004.

14. Angrisani L, Corcione F, Tartaglia A, Tricarico A, Rendano F, Vincenti R, Lorenzo M, Aiello A, Bardi U, Bruni D, Candela S, Caracciolo F, Crafa F, De Falco A, De Werra C, D'Errico R, Giardiello C, Petrillo O, Rispoli G. Cholecystoenteric fistula $(\mathrm{CF})$ is not a contraindication for laparoscopic surgery. Surg Endosc 2001; 15 (9): 1038-1041. Disponible en doi:10.1007/ s004640000317. 